\title{
Familiarity increases the number of remembered Pokémon in visual short-term memory
}

\author{
Weizhen $\mathrm{Xie}^{1}$ • Weiwei Zhang ${ }^{1}$
}

Published online: 8 December 2016

(C) Psychonomic Society, Inc. 2016

\begin{abstract}
Long-term memory (LTM) can influence many aspects of short-term memory (STM), including increased STM span. However, it is unclear whether LTM enhances the quantitative or qualitative aspect of STM. That is, do we retain a larger number of representations or more precise representations in STM for familiar stimuli than unfamiliar stimuli? This study took advantage of participants' prior rich multimedia experience with Pokémon, without investing on laboratory training to examine how prior LTM influenced visual STM. In a Pokémon visual STM change detection task, participants remembered more first-generation Pokémon characters that they were more familiar with than recent-generation Pokémon characters that they were less familiar with. No significant difference in memory quality was found when quantitative and qualitative effects of LTM were isolated using receiver operating characteristic (ROC) analyses. Critically, these effects were absent in participants who were unfamiliar with first-generation Pokémon. Furthermore, several alternative interpretations were ruled out, including general videogaming experience, subjective Pokémon preference, and verbal encoding. Together, these results demonstrated a strong link between prior stimulus familiarity in LTM and visual STM storage capacity.
\end{abstract}

Keywords Short-term memory · Long-term memory · Capacity $\cdot$ Resolution $\cdot$ ROC

Weiwei Zhang

weiwei.zhang@ucr.edu

1 Department of Psychology, University of California, Riverside, 900 University Ave., Riverside, CA 92521, USA
Although a large amount of information can be retained in memory for later retrieval (Brady, Konkle, Alvarez, \& Oliva, 2008), only a small amount of information can be actively maintained over a few seconds to support ongoing tasks (Zhang \& Luck, 2008). While these differences highlight the distinctions between long-term memory (LTM) and short-term memory (STM), these memories also interact with each other. For instance, LTM can influence many aspects of STM, including increased accuracy for familiar verbal materials (e.g., high-frequency words; see Thorn \& Page, 2009, for a review) and visual stimuli (e.g., Buttle \& Raymond, 2003; Curby \& Gauthier, 2007; Curby, Glazek, \& Gauthier, 2009; Sørensen \& Kyllingsbæk, 2012) in STM tasks.

While earlier studies have attributed these LTM benefits to the quantitative aspect (i.e., the total number of retained representations; capacity) of STM (e.g., Chase \& Simon, 1973), some recent studies (e.g., Lorenc, Pratte, Angeloni, \& Tong, 2014; Scolari, Vogel, \& Awh, 2008) seemed to suggest that these LTM effects could instead manifest in the qualitative aspect of STM (i.e., how precise a given STM representation is; resolution). For instance, Scolari et al. (2008) tested visual STM for faces in a change detection task in which participants tried to memorize five faces or cubes and then reported whether a test item was old or new after a short retention interval. Quantitative and qualitative aspects of STM representations were distinguished using manipulations of similarities between memory and test items (Awh, Barton, \& Vogel, 2007; Fukuda, Vogel, Mayr, \& Awh, 2010). When participants memorized faces, a new test item could be a shaded cube (across-category change) or a different face (within-category change). An important assumption in this study is that detection of salient cross-category change does not require precise STM representations, and should be largely constrained by the presence or absence of memory. In contrast, accurate detection of salient within-category change requires both the presence of memory and precise memory representations. Comparable 
change detection performance for upright and inverted faces in the cross-category change condition was thus taken as evidence for similar STM capacities for upright and inverted faces. In contrast, change detection was more accurate for upright faces than inverted faces in the within-category change condition. Because the difference in capacity was already ruled out based on findings in the cross-category change condition, this upright face advantage for detecting withincategory change could only result from the improved resolution for upright faces compared to inverted faces.

However, these selective effects of LTM on STM resolution but not capacity (Lorenc et al., 2014; Scolari et al., 2008) may be limited to faces (more specifically the comparison between upright and inverted faces) and may originate from differences in perceptual encoding instead of memory. That is, improved STM resolution for upright faces compared to inverted faces could simply reflect more efficient perceptual encoding for upright faces (Freire, Lee, \& Symons, 2000; Gao \& Bentin, 2011; Sekuler, Gaspar, Gold, \& Bennett, 2004). Given perceptual effects and memory effects can and should be dissociated (Bae, Olkkonen, Allred, Wilson, \& Flombaum, 2014; Liu \& Chaudhuri, 2000), it is thus unclear whether the previous STM resolution effects can be generalized to visual memory in general, without relying on the comparison between upright and inverted faces.

To test whether existing LTM influences STM quality or quantity, this study uses two novel approaches. The first novel approach was the use of Pokémon (cartoon characters from a series of games, books, TV shows, and movies that are popular in children and adolescences over recent decades) as experimental stimuli. The familiarization procedure for Pokémon (multimedia experience) was entirely different from and independent of the testing procedure used in laboratory tasks, thus avoiding a potential difficulty in determining whether training benefits result from learning of specific procedures or acquired LTM for trained stimuli (Chen, Yee Eng, \& Jiang, 2006). More importantly, this approach took advantage of individual differences in participants' prior multimedia experience with Pokémon instead of investing on laboratory training (and thus avoiding potential issues with insufficient training). Specifically, many participants from the targeted population (college students) were highly familiar (high-familiarity group) with the first-generation Pokémon (released in 1998 when the participants were kindergarteners). In addition, there were also a significant proportion of college students who were less familiar with the first-generation Pokémon (low-familiarity group). ${ }^{1}$ In contrast to these individual differences in familiarity to the first-generation Pokémon, most participants were unfamiliar with the recent-generation Pokémon (e.g., fifth

\footnotetext{
${ }^{1}$ Note, this study was conducted prior to the release of the Pokémon GO game, which featured first-generation Pokémon characters. This was important because it would be difficult to recruit low-familiarity subjects who were not familiar with first-generation Pokémon after the Pokémon $G O$ game became popular.
}

generation Pokémon released in 2011). Consequently, there was a two-way interaction in participants' prior experience with Pokémon. That is, the high-familiarity group was familiar with the first-generation Pokémon, but relatively unfamiliar with the recent-generation Pokémon. In contrast, the low-familiarity group was unfamiliar with Pokémon from both generations. This two-way interaction in participants' prior experience should also manifest in visual STM performance, if prior stimulus familiarity is an important factor for STM task performance. Specifically, STM should be better for first-generation Pokémon relative to the recent-generation Pokémon in the high-familiarity group. In contrast, STM should be comparable for Pokémon of both generations in the low-familiarity group.

In addition, individual differences were also examined to see whether the increases in participants' Pokémon familiarity from the recent-generation to the first-generation could account for increases in STM task performance from the recent-generation to the first-generation. This correlational analysis could reveal the relationship between familiarity and STM task performance without dichotomizing the participants into two groups, and thus preserving statistic power. Both analyses, similar to the comparison of performance between trained and untrained stimuli in previous studies (Chen et al., 2006), can establish whether the effects of LTM memory are specifically linked to participants' prior experience.

The second novel approach is modeling the effects of LTM on quantitative and qualitative aspects of visual STM representation using receiver operating characteristic (ROC) analyses. A ROC curve relates hit rates to false alarm rates according to the signal detection theory (SDT; Wickens, 2001; see Method for details) framework. The quantity and quality of memory representations can be operationalized as the probability that a given item is present in memory $\left(\mathrm{P}_{\mathrm{m}}\right)$ and mnemonic resolution when it is retained, respectively (Zhang \& Luck, 2008). These two aspects can be modeled as distinct components in a ROC curve as detailed in Data Analyses under the "Method" section.

In summary, this study examined effects of Pokémon familiarity on the number and resolution of retained STM representations for Pokémon using ROC analyses. We predict that strong LTM for Pokémon should boost STM performance (in quantity, quality, or both) for the first-generation Pokémon relative to the recent-generation Pokémon, particularly in individuals with higher Pokémon familiarity as compared to individuals with low Pokémon familiarity.

\section{Method}

\section{Participants}

A priori power analysis for a $2 \times 2$ mixed-effect repeatedmeasures ANOVA (Faul, Erdfelder, Buchner, \& Lang, 2009) suggested that a sample size of 22 to 40 participants would 
provide sufficient power for predefined effect sizes $\left(\eta_{\mathrm{p}}{ }^{2}=.04\right.$ $\sim .08$ ) with an $\beta / \alpha$ ratio as 2 and an intercondition correlation as .70. As a result, 30 college students $(19.06 \pm 0.69$ [Mean \pm $\mathrm{SD}$ ] years old, 11 males) were recruited to participate in the study for course credits at University of California, Riverside. Two additional participants were recruited into the study but later excluded from further data analyses because one performed at chance-level (about 50\% of accuracy) and the other only used two options on a six-point confidence scale (see Data Analysis), making the resulting ROC curve difficult to interpret (Yonelinas \& Parks, 2007). All participants had normal color vision and normal (or corrected-to-normal) visual acuity.

\section{Stimulus}

Stimuli were presented on an LCD monitor with a gray background $\left(6.1 \mathrm{~cd} / \mathrm{m}^{2}\right)$ at a viewing distance of $57 \mathrm{~cm}$. Sixty-five unique first-evolution Pokémon characters were selected from www.pokemon.com (Nintendo, Japan), separately for the firstgeneration and the fifth-generation Pokémon (referred to as the recent-generation thereafter), yielding a total of 130 Pokémon characters. These Pokémon characters were comparable in styles, perceptual and conceptual distinctiveness (as defined in Konkle, Brady, Alvarez, \& Oliva, 2010), as well as complexity (see Xie \& Zhang, 2016b, for more details). All Pokémon stimuli were presented in a rectangular area $\left(4.2^{\circ} \times 4.2^{\circ}\right)$, centered at six equally spaced locations on an invisible circle with a radius of $6.5^{\circ}$.

\section{Procedure}

\section{Pokémon change detection task}

As shown in Fig. 1, each trial started with an 800-ms fixation and then a 500-ms memory array of six Pokémon characters that were randomly chosen from either the first-generation or the recent-generation Pokémon set (Pokémon characters were replaced with open-source emojis from http://emojipedia.org/in Fig. 1 for copyright protection of Pokémon images). That is, all memory items on a given trial were either first-generation or recent-generation Pokémon characters. Participants tried to remember all stimuli over a 1,000-ms delay interval. Immediately after the delay, a test array appeared containing one Pokémon stimulus and five $2^{\circ} \times 2^{\circ}$ empty squares at the locations of original memory items. The Pokémon in the test array was the same as the one at the same location in the memory array (i.e., old) on half of trials, and was a different one from the same generation that did not appear in the memory array (i.e., new) on the other half of the trials. Participants reported whether the test item was new or old along with their confidence (sure, probably, or guessing) by clicking on a 6-point confidence scale presented below the test array with a computer mouse. 60 new and 60 old trials were presented for each Pokémon generation, yielding a total of 240 trials, subdivided into 6 blocks. The experimental factor of Pokémon generation (e.g., firstgeneration vs. recent-generation) was randomly mixed within blocks. To suppress verbal encoding, throughout the task participants were required to continuously speak aloud three random digits generated at the beginning of each block.

\section{Pokémon familiarity, preference ratings, naming test, and gaming survey}

Following the STM task, participants used separate 6-point scales to provide subjective ratings on familiarity (from $1=$ unfamiliar to $6=$ familiar) and preference (from $1=$ dislike to $6=$ like) for all 130 Pokémon characters that were sequentially presented in random orders. At the beginning of each trial, a Pokémon character appeared at the center of the computer screen, along with a familiarity scale. Participants provided their familiarity rating to this Pokémon character from $1=$ unfamiliar to 6 = familiar. Subsequently, a likeability scale followed with the Pokémon character remaining on the screen. Participants provided their preference rating to this Pokémon character from $1=$ dislike to $6=$ like. Ten trials for each generation were randomly chosen for an additional multiple-choice naming test to assess participants' verbal memory for these Pokémon stimuli. In this test, participants chose one out of four presented names to match the presented Pokémon character. Their performances were measured as naming accuracy across 10 Pokémon characters for each generation (chance accuracy would be 0.25 ). Last, a survey assessing lifetime gaming experience (hours per day, days per week, and number of years in engagement) across 14 gaming categories (adapted from Kuhn \& Gallinat, 2014) was administered at the end of the experiment. In addition, since a given Pokémon game may belong to more than one gaming categories, we surveyed participants' gaming experience specifically for Pokémon using the same set of questions (hours, days, and years of game playing). Participants proceeded at their own paces and were encouraged to use the rating scales independently.

\section{Data analyses}

Pokémon familiarity As predicted, participants were significantly less familiar, $t(29)=10.60, p<.0001$, with recentgeneration Pokémon (averaged at 1.97 on a 6-point scale), compared to first-generation Pokémon (averaged at 4.65). A median split was thus applied to the familiarity ratings for the first-generation of Pokémon across participants, yielding a group with high familiarity $(5.72 \pm 0.25)$ and a group with significantly lower familiarity $(3.58 \pm 1.35)$ for the firstgeneration Pokémon, $t(28)=5.59, p<.0001$. In contrast, familiarity ratings for the recent-generation Pokémon were not significantly different, $t(28)=1.85, p=.08$, between the highfamiliarity group $(2.30 \pm 1.12)$ and the low-familiarity group $(1.63 \pm 0.82)$, leading to a significant interaction between 


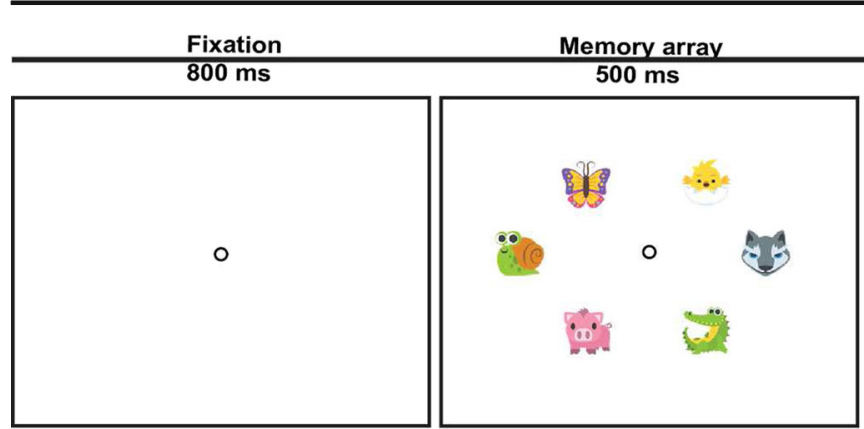

Fig. 1 Illustration of the stimuli and procedure for the Pokémon change detection task. Each trial started with an 800-ms fixation window, followed by a 500-ms memory array of six Pokémon characters from either the first-generation or recent-generation Pokémon sets. After a 1,000-ms delay interval, participants reported whether the Pokémon character in the test array was "new" or "old" as compared to the corresponding

Pokémon generation and subject group, $F(1,28)=11.53, p=$ $.002, \eta_{\mathrm{p}}{ }^{2}=.292$. These two groups did not differ significantly in gender ratio $\left(\chi^{2}<1\right)$ or age, $t(28)=1.06, p=.30$.

Change detection performance Overall change detection performance was measured as Cowan's K, set size $\times$ (hit rate - false alarm rate), which is an estimate of the number of remembered stimuli (Cowan, 2001). Cowan's K at large memory set sizes represents STM storage capacity. A 2 (high-familiarity group vs. low-familiarity group) $\times 2$ (first-generation vs. recentgeneration Pokémon task stimuli) mixed-design repeated-measured ANOVA was performed to examine the differences of Cowan's $\mathrm{K}$ in different conditions (i.e., the median-split approach). In addition, as a complementary test, we calculated the correlation of the differences in Cowan's $\mathrm{K}$ between firstand recent-generation conditions and the differences in familiarity ratings between first- and recent-generation conditions to evaluate whether the increase in Cowan's $\mathrm{K}$ from recentgeneration to first-generation was associated with the increase in Pokémon familiarity (i.e., individual differences approach).

ROC analysis ROC curves were constructed from old versus new responses and associated confidence ratings, separately for each condition (first-generation vs. recent-generation Pokémon task stimuli) and each participant. Different points on ROC curves reflected different levels of decision criteria. The leftmost point on a ROC curve represented the hit rate (proportion of sure-old response when the probed item was old) and false alarm rate (proportion of sure-old response when the probed item was new) at the most conservative decision criterion. The next point on a ROC curve moving rightward represented cumulative hit rate (proportion of sure-old and probably-old response given the probed item was old) and cumulative false alarm rate (proportion of sure-old and probablyold responses given the probed item was new) at a less conservative decision criterion. This procedure was repeated until cumulative hit rates and false alarm rates were aggregated across all confidence levels (Yonelinas \& Parks, 2007).

Empirical ROC patterns were subsequently fitted with theoretical ROC curves from the Zhang and Luck (2008) mixture model using simplex search method (Lagarias, Reeds, Wright, \& Wright, 1998) for each individual at each condition. This ROC mixture model consisted of a high threshold (HT) component $\mathrm{P}_{\mathrm{m}}$ representing the probability of recognizing old items as old, an SDT component $d$ ' representing resolution of noisy STM representation (similar to DeCarlo, 2002), and an additional HT parameter $\mathrm{P}_{\mathrm{n}}$ representing the probability of recognizing nonstudied items as new (i.e., lure rejection; Aly \& Yonelinas, 2012). Cumulative hit rates and false alarm rates from this mixture ROC model could be defined as:

$\mathrm{P}_{(\text {Hit } \mid x>=\mathrm{c})}=\mathrm{P}_{\mathrm{m}} \times \Phi\left(\mathrm{c}-d^{\prime}\right)+\left(1-\mathrm{P}_{\mathrm{m}}\right) \times \Phi(\mathrm{c})$

$\mathrm{P}_{(\mathrm{FA} \mid x>=\mathrm{c})}=\left(1-\mathrm{P}_{\mathrm{n}}\right) \times \Phi(\mathrm{c})$

Here, $\mathrm{P}_{(\mathrm{Hit} \mid x>=\mathrm{c})}$ and $\mathrm{P}_{(\mathrm{FA} \mid \mathrm{x}>=\mathrm{c})}$ represented cumulative hit rates and false alarm rates, respectively, for a response criterion $x$ that was greater or equaled to the confidence level $c$. Note, the inclusion of $\mathrm{P}_{\mathrm{n}}$ for new items is common for modeling perception and STM data (Aly \& Yonelinas, 2012) and helpful for improving model fits. The HT components and the SDT component manifest to different visual aspects of the ROC curves. The HT components produce linear ROC curves (see Fig. 2a), whereas the SDT component produces symmetrical and curvilinear ROC curves (see Fig. 2b). When mixed together (analytically similar to the mixture model of recognition memory; DeCarlo, 2002), the resulting ROC curve is curvilinear and asymmetrical (solid line in Fig. 2c and d). This model can account for all-or-none effects on memory quantity when $d$ ' is at ceiling (see Fig. 1e) as in probabilistic high threshold models (Rouder et al., 2008) or continuous effects on memory quality when $\mathrm{P}_{\mathrm{m}}$ is at ceiling (see Fig. 2f) as in pure SDT models (Wickens, 2001). As a result, the number of retained memory representations $\left(\mathrm{K}_{\mathrm{ind}}\right)$ that is 
independent of mnemonic resolution ( $d^{\prime}$ ') can be estimated as the product of $\left(\mathrm{P}_{\mathrm{m}}+\mathrm{P}_{\mathrm{n}}\right) / 2$ and the number of to-be-remembered stimuli (Wickens, 2001). Mixed-design repeated-measured ANOVAs and correlations were performed on $\mathrm{K}_{\text {ind }}$ and $d$ ', in a similar way as statistical tests on Cowan's K.

In addition to this three-parameter mixture model (with $\mathrm{P}_{\mathrm{m}}$, $\mathrm{P}_{\mathrm{n}}$, and $d^{\prime}$ ), three additional ROC models, including the unequal variance signal detection (UVSD) model (see Parks \& Yonelinas, 2007; Wixted, 2007, for details), dual-process signal detection model (see Yonelinas \& Parks, 2007, for details), and a 2-parameter mixture model (with $\mathrm{P}_{\mathrm{m}}$ and $d$ ') were also fitted to the data. Formal mathematical characterization of UVSD and DPSD can be found in previous studies (see Wixted, 2007; Yonelinas \& Parks, 2007, for details). The two two-parameter mixture model is essentially the threeparameter mixture model with $\mathrm{P}_{\mathrm{n}}$ fixed at zero. Formal model comparisons were performed for all four models to determine the best-fit model for the empirical ROC data.

\section{Results and discussion}

\section{Cowan's K}

Differences in Pokémon familiarity across Pokémon generations and subject groups also manifested in Cowan's K (see Fig. 3a) in that participants had higher Ks for first-generation than recent-generation Pokémon in the high-familiarity group, $t(14)=3.47, p=.004$, Cohen's $d=.90$, but not in the lowfamiliarity group $(t<1)$, yielding a significant two-way interaction, $\left.F(1,28)=6.40, p=.017, \eta_{\mathrm{p}}{ }^{2}=.186\right)$. In addition, there was a significant main effect of Pokémon generation, $F(1,28)=$ $6.73, p=.015, \eta_{\mathrm{p}}{ }^{2}=.194$, indicating that more first-generation than recent-generation Pokémon characters were retained in STM. In contrast, there was no significant main effect of subject groups, $F(1,28)=1.54, p=.23, \eta_{p}{ }^{2}=.052$, suggesting the two groups had comparable STM capacity in general.

A stronger test of the relation between familiarity and STM capacity was to examine whether the increase in $\mathrm{K}$ and familiarity from recent-generation to first-generation Pokémon associated with each other or not. Indeed, participants who were more familiar with first-generation relative to recentgeneration Pokémon tended to remember more first- (vs. recent-) generation Pokémon $(r=.51,95 \%$ CI $[.18, .73], p=$ .004; see Fig. 3b).

\section{ROC analyses}

$K_{\text {ind }}$ and $d^{\prime}$

To identify whether memory quantity, quality, or both drove these effects, we examined parameters from the mixture model, which provided good overall fits $\left(R^{2}\right.$ adjusted $\left.\geq 99 \%\right)$. The ROC curves were clearly dissociable between the first-generation and recentgeneration Pokémon for the high-familiarity group (see Fig. 4a), but not for the low-familiarity group (see Fig. 4b), in line with the significant difference in Cowan's K as previously shown. This pattern seemed to largely result from the $K_{\text {ind }}$ (see Fig. 4c), in that participants remembered more first-generation (vs. recent-generation) Pokémon in the high-familiarity group, $t(14)=3.05, p=$ .009 , Cohen's $d=.79$, but not in the low-familiarity group, $t(14)=$ $1.28, p=.22$, Cohen's $d=.33$, leading to a significant interaction, $F(1,28)=8.87, p=.006, \eta_{\mathrm{p}}{ }^{2}=.241$, although none of the main effects was significant (Pokémon generation: $F(1,28)=1.12, p=$ .30 ; subject group: $F(1,28)=1.80, p=.19)$. In comparison, no significant effect was observed for resolution $(d$ ', see Fig. 4d; Pokémon generation: $F(1,28)=1.41, p=.25$; subject group: $F(1,28)=2.52, p=.12$; interaction: $F<1)$.

Again, participants who were more familiar with firstgeneration relative to recent-generation Pokémon tended to remember more first- (vs. recent-) generation Pokémon (see Fig. 4e, $r=.49,95 \%$ CI $[.16, .72], p=.006$. This relationship did not manifest in STM resolution (see Fig. 4f, $r=.05,95 \%$ CI $[-.32, .40], p=.81)$, yielding a significantly lower resolution effect than the capacity effect $(z=1.69, p=.045$, one-tailed) based on a test on correlated correlations (Meng, Rosenthal, \& Rubin, 1992).

Separate analyses on $P_{m}$ and $P_{n}$

Similar results as $\mathrm{K}_{\text {ind }}$ - which combined $\mathrm{P}_{\mathrm{m}}$ and $\mathrm{P}_{\mathrm{n}}$-were also found for $\mathrm{P}_{\mathrm{m}}$, but not for $\mathrm{P}_{\mathrm{n}}$, when separate ANOVAs were performed on $\mathrm{P}_{\mathrm{m}}$ and $\mathrm{P}_{\mathrm{n}}$. The probability of recognizing old items as old $\left(\mathrm{P}_{\mathrm{m}}\right)$ yielded a similar pattern as $\mathrm{K}_{\text {ind. }}$. There was a significant interaction between Pokémon generation and subject group on $\mathrm{P}_{\mathrm{m}}, F(1,28)=7.10, p=.013, \eta_{\mathrm{p}}{ }^{2}=.202$, accompanied by a significant main effect of Pokémon generation, $F(1,28)=7.67, p=.010, \eta_{\mathrm{p}}{ }^{2}=.125$. The main effect of subject group, $F(1,28)=3.85, p=.060, \eta_{\mathrm{p}}{ }^{2}=.121$, was ambiguous. Nonetheless, the current conclusion would not critically depend on the statistical significance of this main effect of subject group on $\mathrm{P}_{\mathrm{m}}$. More importantly, as participants' familiarity with first-generation Pokémon relative to recent-generation Pokémon increased, they were indeed more likely to remember first-generation Pokémon relative to recent-generation Pokémon when the test items were from memory $\left(\mathrm{P}_{\mathrm{m}}, r=.54,95 \% \mathrm{CI}[.22, .75], p=.002\right)$. In contrast, no systematic effect was found for $\mathrm{P}_{\mathrm{n}}$. That is, none of the main effect of Pokémon generation $(F<1)$, the main effect of subject group $(F<1)$, or the interaction effect between these two variables, $F(1,28)=1.11, p=.30, \eta \mathrm{p}^{2}=.038$, was significant for $\mathrm{P}_{\mathrm{n}}$. No significant correlation was found between the difference in familiarity between the first- and recent-generation Pokémon and the difference in $\mathrm{P}_{\mathrm{n}}$ across generations ( $r=.17,95 \%$ CI $[-.19, .50], p=.34)$.

Although $\mathrm{P}_{\mathrm{m}}$ and $\mathrm{P}_{\mathrm{n}}$, along with $d$, were simultaneously extracted from ROC data, they were largely driven by 

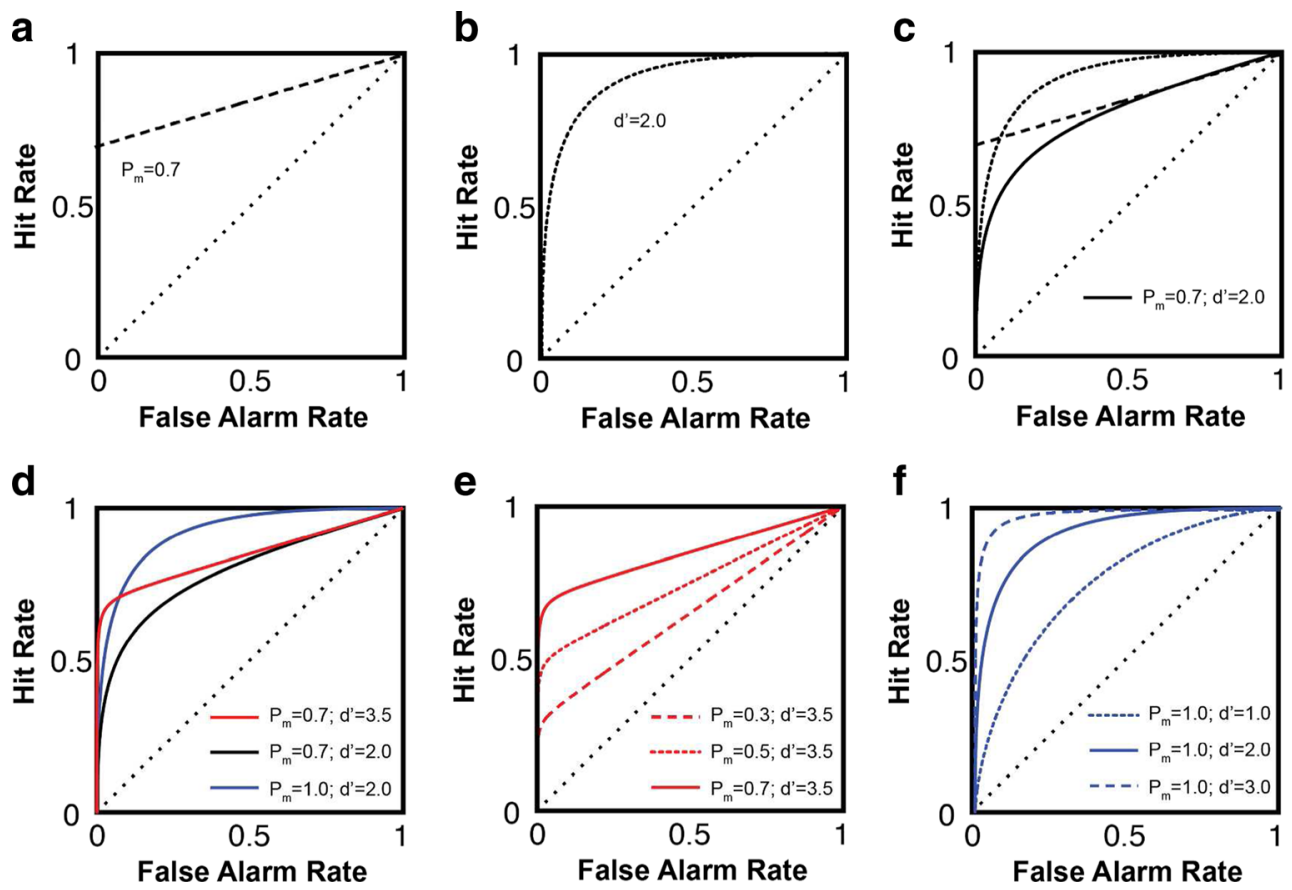

Fig. 2 Implementation of Zhang and Luck (2008) visual STM mixture model for ROCs. Visual STM can be modeled with two distinct components, (a) a high threshold (HT) component (linear ROC, $\mathrm{P}_{\mathrm{m}}$ represents the probability that a given stimulus is retained in STM) and (b) a SDT component (symmetrical and curvilinear ROC, $d$ ' represents mnemonic quality/resolution of noisy memory representations). When mixed together (c), the resulting ROC is curvilinear and asymmetrical. $\mathbf{d}$ The mixture

model can approach HT model as $d^{\prime}$ increases (e.g., increasing linearity from the black line to red line) or SDT models as $\mathrm{P}_{\mathrm{m}}$ increases (e.g., increasing symmetry from the black line to blue line). Consequently, the mixture model can account for pure HT ROCs (e, e.g., Rouder et al., 2008) and SDT ROCs (f, e.g., Wickens, 2001) for visual STM (Color figure online).

participants' responses from different trials. $\mathrm{P}_{\mathrm{m}}$ was based on trials where the Pokémon in the test array was the same as one of the Pokémon in the memory array. In contrast, $\mathrm{P}_{\mathrm{n}}$ was based on trials where the Pokémon in the test array was different from Pokémon in the memory array. More importantly, they reflected different memory processes. $\mathrm{P}_{\mathrm{m}}$ reflected the

probability that an old Pokémon was correctly recognized as old. It was largely determined by the probability that the tested item was encoded in STM. However, $\mathrm{P}_{\mathrm{n}}$ reflected lure rejection, the probability that a new Pokémon was correctly recognized as new. Given that $\mathrm{P}_{\mathrm{m}}$ and $\mathrm{P}_{\mathrm{n}}$ can be distorted by participants' biases in reporting old and new responses, respectively,

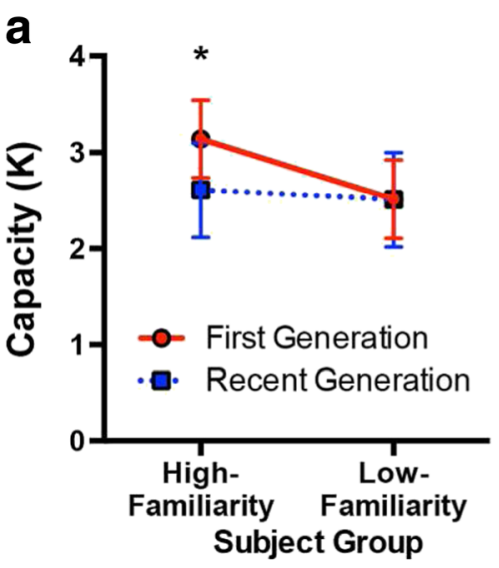

b

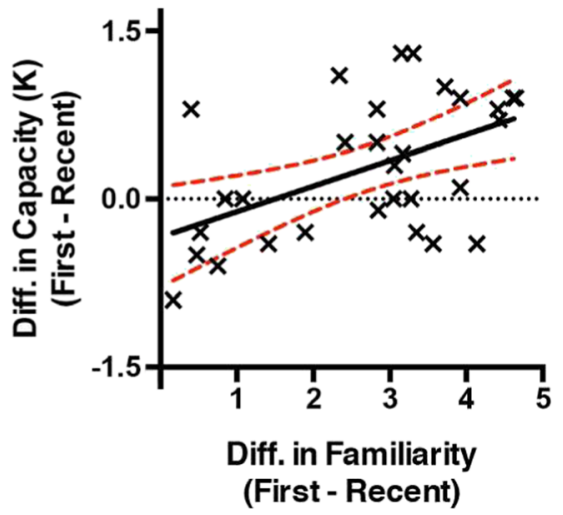

Fig. 3 Results in Cowan's K (a) and its relationship with familiarity (b). a High-familiarity group remembered more (larger Cowan's K) firstgeneration Pokémon characters relative to recent-generation Pokémon, whereas low-familiarity group remembered similar numbers of Pokémon characters across the two generations. Error bars in a represent within-subject $95 \%$ confidence intervals (Morey, 2008), $* p<.05$. b

Across participants, differences in Cowan's $\mathrm{K}$ between first and recent Pokémon generations correlated significantly with differences in Pokémon familiarity between Pokémon generations. b The solid and broken lines represented linear regression fit and its 95\% confidence intervals, respectively (Color figure online). 
a

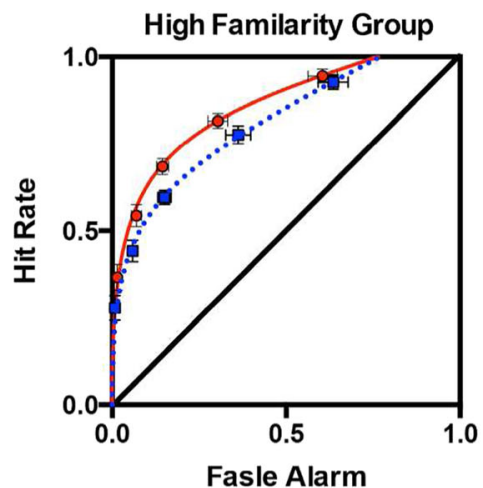

\section{C}

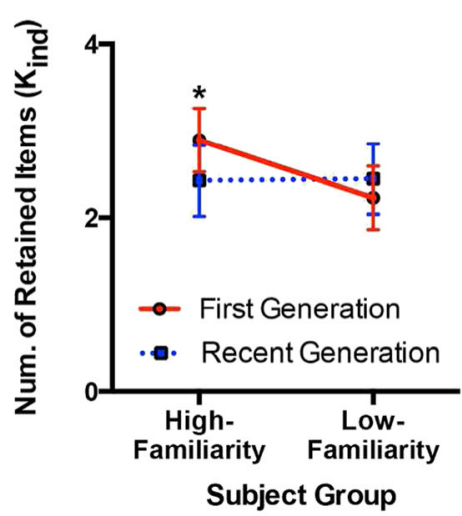

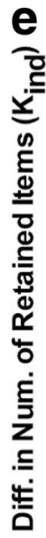

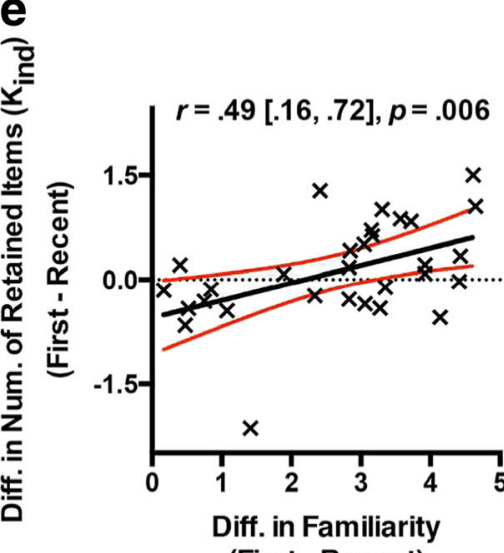

(First - Recent)

Fig. 4 ROC results. Observed ROCs for the high-familiarity group (a) and low-familiarity group (b). The vertical and horizontal error bars in a and $\mathbf{b}$ indicate standard errors of hit rates and false alarm rates, respectively. The two components of the mixture model estimated from ROCs: Number of retained items $\left(\mathrm{K}_{\text {ind }}=\mathrm{K}\right.$ independent of resolution, $\left.\mathbf{c}\right)$ and resolution $\left(d^{\prime}, \mathbf{d}\right)$ across subject groups and Pokémon generations. Error bars in $\mathbf{c}$ and $\mathbf{d}$ represent within-subject $95 \%$ confidence intervals

the compound measure $\mathrm{K}_{\mathrm{ind}}$ is a more robust estimate of STM storage capacity.

\section{Alternative ROC models}

We compared model fits for each participant under each condition using Akaike information criterion (AIC). Smaller AIC value indicates a better model fit. As shown in Fig. 5, the

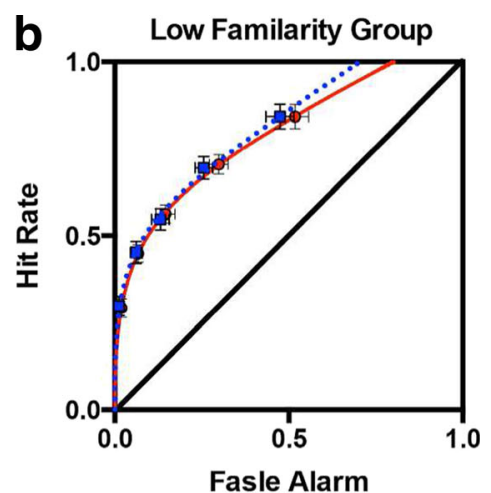

d

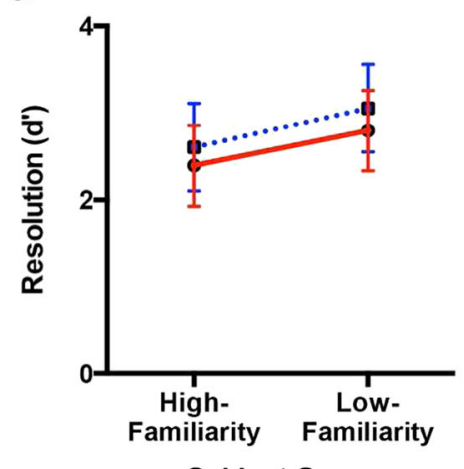

Subject Group

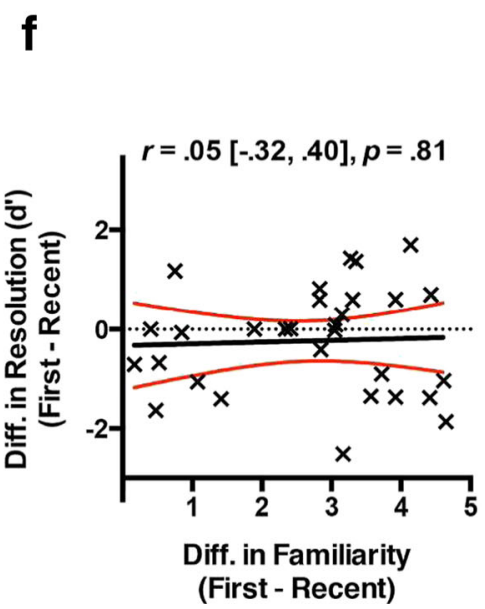

(Morey, 2008), $* p<.05$. The relationship between STM and familiarity $(\mathbf{e} \& \mathbf{f})$. Across participants, differences in the number of retained items $\left(\mathrm{K}_{\text {ind }}, \mathbf{e}\right)$, but not in resolution $\left(d^{\prime}, \mathbf{f}\right)$, between first- and recent-generation of Pokémon correlated significantly with differences in Pokémon familiarity between Pokémon generations. In $\mathbf{e}$ and $\mathbf{f}$, the solid and broken lines respectively represent linear regression fit and its $95 \%$ confidence intervals (Color figure online).

three-parameter mixture model, in general, yielded smaller AIC values across conditions in the majority of participants, as compared to the two-parameter mixture model without $P_{n}$ (three-parameter mixture model wins 60 out of 60 times, $\Delta \mathrm{AIC}_{\text {mean }}=-2.53$, see Fig. 5a), the UVSD model (three-parameter mixture model wins 51 out of 60 times $\Delta \mathrm{AIC}_{\text {mean }}=$ 2.46, Fig. 5b), and the DPSD model (three-parameter mixture model wins 47 out of 60 times, $\Delta \mathrm{AIC}_{\text {mean }}=-1.83$, Fig. $5 \mathrm{c}$ ). 
Additional model selection was performed for each participant at each condition using Akaike weights (see Wagenmakers \& Farrell, 2004, for details). Akaike weights from individual subjects (see Fig. 6a and c) and group average (see Fig. 6b and d) suggested that the three-parameter mixture model reliably outperformed all other three models. Specifically, the three-parameter mixture model yielded weights of $40 \%$ to $50 \%$ across two experimental conditions, which was 2 to 3 times more likely to be the best-fit model compared with other models (weighted around $15 \%$ to $25 \%$ on average). Together, these formal model comparisons provide evidence supporting the three-parameter mixture model over other competing models that were commonly tested in the recognition memory literature (Yonelinas \& Parks, 2007).

\section{Factors beyond familiarity}

\section{Gaming experience}

We further ruled out alternative explanations that factors other than LTM familiarity (a proxy for phenomenological LTM), such as gaming experience, preference, and verbal encoding could account for the present findings on capacity. Although lifetime gaming time for Pokémon provided a more objective assessment of participants' overall Pokémon gaming experience for all generations of Pokémon, this measure was less selective because it did not reflect participants' different experiences with first-generation and recent-generation Pokémon. In addition, it did not assess participants' prior experience with Pokémon through other multimedia experiences, such as Pokémon books, TV episodes, and movies. Nonetheless, lifetime Pokémon gaming time significantly correlated with subjective familiarity ratings (first-generation: Spearman $r=.76,95 \%$ CI $[.51, .90], p<.0001$; recent-generation: Spearman $r=.49,95 \%$ CI [.09, .77], $p=.005)$. In addition, high-familiarity group (vs. low-familiarity) group had significantly longer Pokémon gaming time $(z=3.19, p$ $=.0014$, Mann-Whitney $U$ test). More importantly, lifetime Pokémon gaming time significantly correlated with Cowan's $\mathrm{K}$ (Spearman $r=.42,95 \%$ CI $[.05, .71], p=.020)$ and $\mathrm{P}_{\mathrm{m}}$ (Spearman $r=.61,95 \%$ CI $[.32, .80], p<.001$ ), for firstgeneration Pokémon, but not for recent-generation Pokémon (Cowan's K: Spearman $r=.29,95 \%$ CI [-.09, .60], $p=.12$; $\mathrm{P}_{\mathrm{m}}$ : Spearman $r=.21,95 \%$ CI $\left.[-.16, .53], p=.27\right)$. No significant correlation was found between Pokémon gaming time and $d^{\prime}$ 'or between Pokémon gaming time and $\mathrm{P}_{\mathrm{n}}$ for either Pokémon generation (All $p \mathrm{~s}>.25$ ).

Given that Pokémon familiarity correlated with Pokémon gaming experience, could overall gaming experience instead of Pokémon familiarity cause the observed effects in STM? This alternative interpretation seems plausible because intensive video gaming experience can enhance various aspects of perception and cognition (see reviews from Granic, Lobel, \& a

3-Parameter "Slot" Model vs. 2-Parameter "Slot" Model

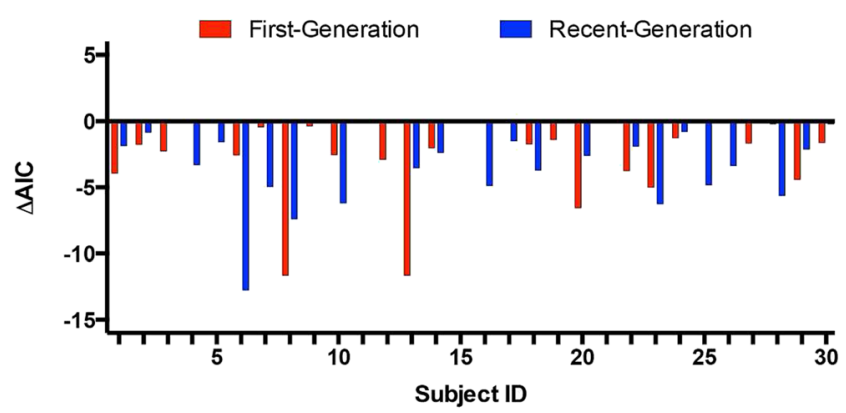

b

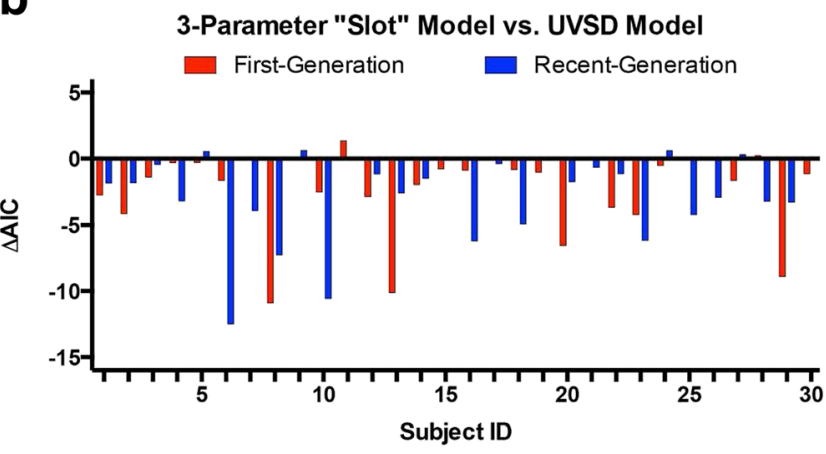

C

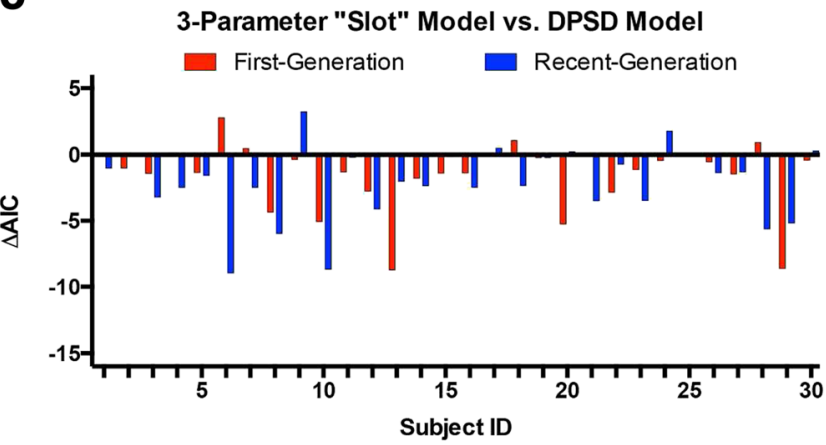

Fig. 5 Model comparison for three-parameter $\left(\mathrm{P}_{\mathrm{m}}, \mathrm{P}_{\mathrm{n}}\right.$, and $\left.d^{\prime}\right)$ mixture model over two-parameter $\left(\mathrm{P}_{\mathrm{m}}, d^{\prime}\right)$ mixture model (a), UVSD model (b), and DPSD model (c). Differences in Akaike information criterion ( $\triangle \mathrm{AIC}$ ) between the mixture model and one of the alternate models were plotted on the $y$-axis for each participant under each experimental condition (first-generation vs. recent-generation task stimuli). A negative $\Delta \mathrm{AIC}$ value means better fit of the three-parameter mixture model over the alternative model (Color figure online).

Engels, 2014; Green \& Bavelier, 2012), including visual STM (e.g., Blacker, Curby, Klobusicky, \& Chein, 2014). It is thus pivotal to assess the effects of general video gaming experience on visual STM. Therefore, Mann-Whitney $U$ test and Spearman correlation were respectively used to examine group differences in lifetime gaming scores and their correlations with other variables (see Table 1). It showed that individual with more prior Pokémon familiarity also spent significantly longer gaming time on four categories of video games (online role-play, click-and-point adventure, action adventure, and logic/puzzle). In addition, lifetime Pokémon gaming time 

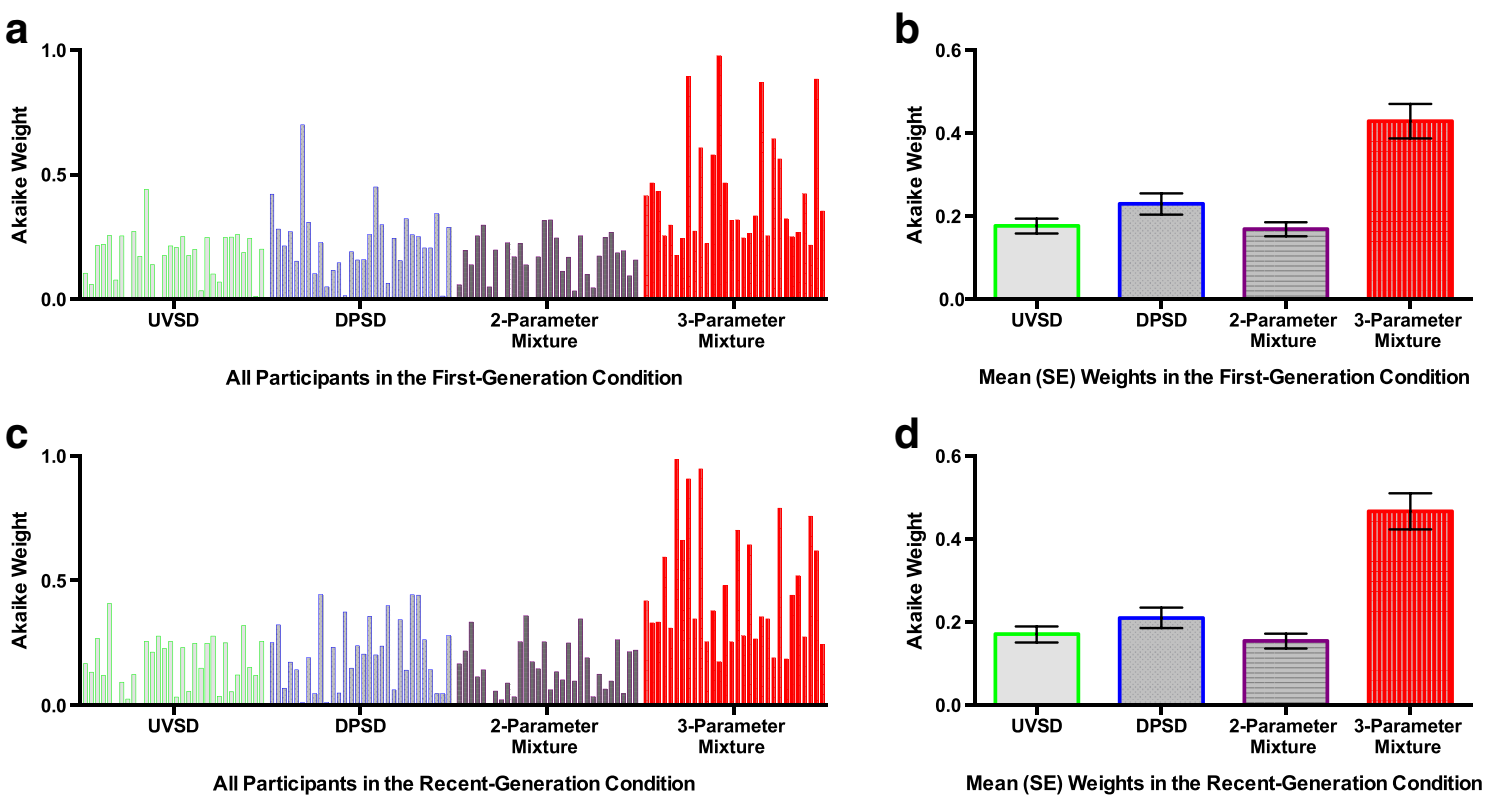

Fig. 6 Akaike weights for individual subjects (a \& c) and group average (b \& d) of the four models (i.e., UVSD, DPSD, two-parameter mixture, and three-parameter mixture, plotted in different colors) in the firstgeneration Pokémon condition $(\mathbf{a} \& \mathbf{b})$ and the recent-generation Pokémon condition $(\mathbf{c} \& \mathbf{d})$. Each bar in a and $\mathbf{c}$ represents an Akaike weight of a given model for data from one participant, sorted based on

significantly correlated with lifetime gaming time for those four game categories (see Table 1). If overall gaming experience, instead of specific Pokémon experience, produced the observed quantity enhancement in STM, gaming time should also predict STM capacity (Cowan's K or $\mathrm{P}_{\mathrm{m}}$ ) for recentgeneration Pokémon. However, none of these correlations reached significance (all $p \mathrm{~s}>.10$ ). In addition, the observed boost in STM storage capacity was specific to first-generation Pokémon, ruling out the alternative account based on the overall gaming experience.

\section{Subjective preference}

Subjective preference for Pokémon showed a significant main effect of generation, $F(1,28)=32.88, p<.001, \eta_{\mathrm{p}}{ }^{2}=.540$, suggesting that participants in general liked first-generation Pokémon better (first- vs. recent-generation: $3.95 \pm 0.70$ vs. $3.04 \pm 0.64)$. However, there was no significant main effect of subject group $(F<1)$ or interaction between Pokémon and subject group $(F<1)$, indicating preference could not account for the interaction effect in STM performance.

\section{Verbal encoding}

Pokémon naming test showed a significant interaction effect, $F(1,28)=4.88, p=.036, \eta_{\mathrm{p}}{ }^{2}=.148$, similar to STM performance. Specifically, participants in the high familiarity group named first-generation over recent-generation Pokémon more

subject numbers (1 to 30). Error bars in the group average (b \& d) represent standard errors of the weights. Overall, the three-parameter mixture model provides the best account for the observed data among all four models in that it is about 2 to 3 times more likely to be the best-fit model compared with the other three models (Wagenmakers \& Farrell, 2004) (Color figure online)

accurately (accuracy: $0.76 \pm 0.17$ vs. $0.49 \pm 0.22), t(14)=$ 5.73, $p<.001$, Cohen's $d=1.48$, compared with those in the low familiarity group (accuracy: $0.51 \pm 0.21$ vs. $0.41 \pm$ $0.22), t(14)=1.91, p=.075$. This seemed to suggest that strategic verbal encoding might have led to the observed effects on visual STM. We found this alternative interpretation unlikely for two reasons. First, participants performed the STM task with a verbal suppression task that minimized verbal encoding (Avons \& Phillips, 1980; Cowan, 2001; Jackson \& Raymond, 2008). Second, the increase in Pokémon naming accuracy for first-generation over recent-generation Pokémon was not significantly predictive of the boost in visual STM in Cowan's K or $\mathrm{K}_{\text {ind }}$ (all $p \mathrm{~s}>.05$ ).

\section{General discussion}

This study tested how individual differences in prior multimedia experience with Pokémon affected the number and quality of remembered Pokémon characters in STM. A ROC mixture model was developed to decompose overall ROCs from a Pokémon change detection task into an SDT component $\left(d^{\prime}\right)$ representing the resolution of noisy STM representations and HT components representing the number of retained items that is independent of resolution $\left(\mathrm{K}_{\mathrm{ind}}\right)$. We found that one group of participants remembered more (larger Cowan's K) firstgeneration Pokémon characters that they were more familiar with than recent-generation Pokémon that they were less 
Table 1 Participants' lifetime gaming experience across different game categories

\begin{tabular}{|c|c|c|c|c|c|c|c|c|}
\hline \multirow{3}{*}{$\begin{array}{l}\text { Lifetime gaming experience in hours } \\
\text { (hours } \times \text { days per week } \times 52 \times \text { years) }\end{array}$} & \multirow{2}{*}{\multicolumn{2}{|c|}{$\frac{\text { High familiarity }}{(n=15 ; \mathrm{M}: \mathrm{F}=6: 9)}$}} & \multirow{2}{*}{\multicolumn{2}{|c|}{$\frac{\text { Low familiarity }}{(n=15 ; \mathrm{M}: \mathrm{F}=5: 10)}$}} & \multirow[t]{3}{*}{$z^{\mathrm{b}}$} & \multirow[t]{3}{*}{$p$} & \multirow{3}{*}{$\begin{array}{l}r_{\text {Spearman }} \text { with Pokémon } \\
\text { experience }^{\mathrm{c}}\end{array}$} & \multirow[t]{3}{*}{$p$} \\
\hline & & & & & & & & \\
\hline & Mean & $\mathrm{SD}$ & Mean & SD & & & & \\
\hline Pokémon & 2846.1 & 4019.9 & 459.3 & 885.9 & $3.19 * *$ & .001 & & \\
\hline Building games & 256.5 & 526.1 & 268.7 & 683.9 & -0.42 & .67 & .06 & .77 \\
\hline Simulation games & 427.3 & 665.1 & 322.4 & 821.8 & 1.41 & .16 & .26 & .16 \\
\hline Racing games & 837.2 & 2631.8 & 162.9 & 420.1 & 1.31 & .19 & .33 & .07 \\
\hline Sports games & 651.7 & 1802.3 & 91.9 & 209.6 & 0.69 & .49 & .06 & .75 \\
\hline Online role play & 1889.3 & 3409.6 & 1293.1 & 4880 & $2.60 * *$ & .009 & $.54 * *$ & .002 \\
\hline Single-player role play & 984.5 & 2139.7 & 263.5 & 815.2 & 1.8 & .072 & .31 & .10 \\
\hline Action-based role play & 409.9 & 1084.3 & 540.8 & 1871.7 & 0.97 & .33 & .13 & .50 \\
\hline Click-and-point adventure & 31.2 & 70.3 & $\mathbf{0}$ & - & $2.07 *$ & .038 & $.41 *$ & .026 \\
\hline Action adventures & 2483.9 & 4371.5 & 142.1 & 414.4 & $3.06 * *$ & .001 & $.51 * *$ & .004 \\
\hline Platform games & 949.9 & 1522.7 & 187.2 & 338 & 1.46 & .14 & .33 & .074 \\
\hline Ego shooter & 578.9 & 1959.1 & 781 & 2181.4 & 0.11 & .91 & .23 & .22 \\
\hline Third-person shooter & 482.7 & 722.6 & 2742.1 & 9341.3 & 0.56 & .57 & .21 & .26 \\
\hline Logic/puzzle & 358.8 & 429.1 & 79.7 & 153.3 & $2.34 *$ & .019 & $.37 *$ & .044 \\
\hline Arcade games & 214.2 & 415.8 & 83.2 & 200.2 & 1.11 & .27 & .11 & .55 \\
\hline
\end{tabular}

Note. Participants were grouped based on their subjective ratings of phenomenological familiarity to first-generation Pokémon. Mann-Whitney $U$ test was used to examine group differences in gaming experience, given the lack of normality. Gaming categories with statistically significant group difference were bolded in the table. Spearman's rank-order correlation of gaming experience for specific gaming categories with Pokémon gaming experience. $\mathrm{M}=$ Male, $\mathrm{F}=$ Female

$* p<.05 . * * p<.01$

familiar with. In addition, this overall boost in STM compound capacity resulted from an increase in the number of retained STM representations, instead of the mnemonic resolution, from ROC analyses. In contrast, these effects were absent in another group of participants who were less familiar with Pokémon of both generations. Furthermore, across participants, the increase in Pokémon familiarity from recentgeneration to first-generation significantly correlated with the increase in STM storage capacity (Cowan's $\mathrm{K}$ and $\mathrm{K}_{\text {ind }}$ ), but not with the change in mnemonic resolution $\left(d^{\prime}\right)$, from recent-generation to first-generation. Critically, these findings were unlikely to be attributed to gaming experience, subjective preference, or verbal encoding. Together, these results suggested that existing LTM could selectively boost visual STM storage capacity with little effect on STM resolution.

This study has generalized previous findings that LTM boosts STM storage capacity from verbal memories to visual memories, which is not trivial (Luck, 2008). One of the major differences between verbal and visual memories is that verbal stimuli tend to have stronger and more direct structural mappings between semantic representations in LTM and those in STM. That is, encoding a word to semantic level in STM, by definitions, also activates corresponding semantic representations in LTM. Consequently, the heightened activation from LTM could boost STM in return (Oberauer \& Lange, 2009).
In contrast, this interaction seems less robust in visual memory (Luck, 2008) in that some previous studies have failed to demonstrate significant LTM effects in the visual domain (e.g., Chen et al., 2006; Huang, 2011; Olson \& Jiang, 2004; Pashler, 1988). These null results, however, could stem from weak LTM traces due to insufficient training (Olson \& Jiang, 2004).

Stronger LTM traces could potentially account for the present and some of the previous significant effects of LTM on STM for "specialized" stimuli, such as faces (e.g., Buttle \& Raymond, 2003; Curby \& Gauthier, 2007; Jackson \& Raymond, 2008) and objects of expertise (e.g., Curby et al., 2009; Moore, Cohen, \& Ranganath, 2006; Wagar \& Dixon, 2005). Processing of these stimuli is highly developed in humans to the extent that dedicated neural substrates (e.g., fusiform face area; Grill-Spector, Knouf, \& Kanwisher, 2004) or computational mechanisms (e.g., holistic processing; McKone, Kanwisher, \& Duchaine, 2007), though still under debate (e.g., Gauthier, Tarr, Anderson, Skudlarski, \& Gore, 1999), may be recruited. These specialized mechanisms may also support recognition of non-face objects, with acquired expertise (Gauthier et al., 1999; Rezlescu, Barton, Pitcher, \& Duchaine, 2014), including Pokémon stimuli used in the present study (James \& James, 2013). Specifically, the initially novel and artificial Pokémon characters may elicit 
robust activation in fusiform areas after acquired familiarization (James \& James, 2013). With these "specialized" stimuli, it is possible that both STM and LTM reactivate corresponding sensory cortices at encoding and retrieval (Jonides et al., 2008), making interactions between STM and LTM possible (Ranganath, 2004). More importantly, existing LTM for these stimuli of exceeding expertise may be strong enough to elicit automatic link between existing LTM representations and STM (Beck \& van Lamsweerde, 2011). Distinctive from these previous findings, the present study contributes to the literature by distinguishing the influence of LTM on the quantitative and qualitative aspects of STM, and further attributes the boost in STM performance to capacity.

This capacity account seems at odds with two previous findings that LTM sharpens STM quality (Lorenc et al., 2014; Scolari et al., 2008), which may be an artifact of the comparison of STM for upright and inverted faces. This alternative interpretation is unlikely to account for the present findings because the capacity effects in the present study are linked to the difference in participants' familiarity for Pokémon, without relying on comparisons of upright and inverted stimuli. Thus, the present capacity effects are more likely to reside in STM instead of perception and more generalizable to visual cognition than the previous resolution effects.

This study has developed a quantitative method for estimating STM capacity and resolution. Although this model is an extension of Zhang and Luck's (2008) mixture model from recall to recognition (change detection), it is practically more flexible than delayed estimation performance, which is limited to features in circular feature space (e.g., color, orientation, closed-contour, face). The ROC mixture model can be applied to any feature dimensions, any combinations of these features, or complex stimuli. Note, the reliability of the ROC method is established by fitting simulated data using a wide range of parameters with the resulting parameters successfully matching the parameters used in simulation (Xie \& Zhang, 2016a).

It is important to further establish psychological meanings of the HT and SDT components (Xie \& Zhang, 2016a). First, if the ROC parameters represent quantitative and qualitative aspects of STM storage, they should correlate with the corresponding parameters from Zhang and Luck's (2008) mixture model for delayed estimation data. Second, the ROC parameters should be selectively affected by experimental manipulations that selectively affect STM capacity or resolution, similar to experimental dissociation of Zhang and Luck's (2008) mixture model parameters (Zhang \& Luck, 2008). For example, different amount of encoding time using visual STM consolidation masking (detailed in Vogel, Woodman, \& Luck, 2006) that affects the number, but not the resolution, of encoded STM representations (Zhang \& Luck, 2008) should have similar effects on $\mathrm{K}_{\text {ind }}$ from the ROC mixture model in the change detection paradigm. In contrast, white noise added to the memory array that significantly affects mnemonic resolution, but not the number, of representations in visual STM (Zhang \& Luck, 2008) should lead to similar changes in $d$ ' from the ROC mixture model in the change detection paradigm. These predictions need to be tested in future studies.

It is pivotal to note that LTM can improve STM task performance through mechanisms other than increased storage capacity. First, STM performance can be boosted by chunking multiple pieces of information into a singular representation (Cowan, 2001). Second, existing LTM could bias STM encoding to focus more on distinctive information (Olsson \& Poom, 2005). Third, LTM can facilitate various processes in STM, including consolidation, maintenance, retrieval, and executive control (Thorn \& Page, 2009). Fourth, statistical regularity acquired over time could increase the amount of information retained in STM with more efficient encoding (Olson, Jiang, \& Moore, 2005). Future research thus needs to elucidate relationships between these factors and the present capacity effect.

\section{Conclusion}

This study has provided some novel evidence supporting the effects of existing LTM on STM storage capacity. Specifically, differences in prior stimulus familiarity across stimuli and participants could account for differences in STM capacity. That is, participants with higher familiarity with the first-generation Pokémon could remember more first-generation Pokémon characters than recent-generation characters in visual STM. In contrast, participants who were unfamiliar with both Pokémon generations remembered similar numbers of Pokémon characters from both Pokémon generations. These results provided some support for the relationship between existing LTM (prior familiarity) and STM storage capacity. Future research needs to explore possible mechanism(s) underlying these capacity effects.

Acknowledgements This study was made possible by a start-up grant from University of California, Riverside to Weiwei Zhang. We thank Gavin Zhang and Kyra Zhang for inspiring us to use Pokémon characters in the study and thank Jonathan Caplan for assistance in data collection. We also would like to thank Nancy Carlisle and another anonymous reviewer for several excellent suggestions regarding the manuscript.

\section{References}

Aly, M., \& Yonelinas, A. P. (2012). Bridging consciousness and cognition in memory and perception: Evidence for both state and strength processes. PLoS ONE, 7(1), e30231. doi:10.1371/journal. pone. 0030231

Avons, S. E., \& Phillips, W. A. (1980). Visualization and memorization as a function of display time and poststimulus processing time. Journal of Experimental Psychology: Human Learning and Memory, 6(4), 407-420. 
Awh, E., Barton, B., \& Vogel, E. K. (2007). Visual working memory represents a fixed number of items regardless of complexity. Psychological Science, 18(7), 622-628. doi:10.1111/j.14679280.2007.01949.x

Bae, G. Y., Olkkonen, M., Allred, S. R., Wilson, C., \& Flombaum, J. I. (2014). Stimulus-specific variability in color working memory with delayed estimation. Journal of Vision, 14(4), 7. doi:10.1167/14.4.7

Beck, M. R., \& van Lamsweerde, A. E. (2011). Accessing long-term memory representations during visual change detection. Memory \& Cognition, 39(3), 433-446. doi:10.3758/s13421-010-0033-4

Blacker, K. J., Curby, K. M., Klobusicky, E., \& Chein, J. M. (2014). Effects of action video game training on visual working memory. Journal of Experimental Psychology: Human Perception and Performance, 40(5), 1992-2004. doi:10.1037/a0037556

Brady, T. F., Konkle, T., Alvarez, G. A., \& Oliva, A. (2008). Visual longterm memory has a massive storage capacity for object details. Proceedings of the National Academy of Sciences of the United States of America, 105(38), 14325-14329. doi:10.1073 /pnas.0803390105

Buttle, H., \& Raymond, J. E. (2003). High familiarity enhances visual change detection for face stimuli. Perception \& Psychophysics, 65(8), 1296-1306.

Chase, W. G., \& Simon, H. A. (1973). Perception in chess. Cognitive Psychology, 4(1), 55-81.

Chen, D., Yee Eng, H., \& Jiang, Y. (2006). Visual working memory for trained and novel polygons. Visual Cognition, 14(1), 37-54. doi:10.1080/13506280544000282

Cowan, N. (2001). The magical number 4 in short-term memory: A reconsideration of mental storage capacity. Behavioral and Brain Sciences, 24(1), 87-185. doi:10.1177/0963721409359277

Curby, K. M., \& Gauthier, I. (2007). A visual short-term memory advantage for faces. Psychonomic Bulletin \& Review, 14(4), 620-628. doi:10.3758/BF03196811

Curby, K. M., Glazek, K., \& Gauthier, I. (2009). A visual short-term memory advantage for objects of expertise. Journal of Experimental Psychology: Human Perception and Performance, 35(1), 94-107. doi:10.1037/0096-1523.35.1.94

DeCarlo, L. T. (2002). Signal detection theory with finite mixture distributions: Theoretical developments with applications to recognition memory. Psychological Review, 109(4), 710-721. doi:10.1037 //0033-295X.109.4.710

Faul, F., Erdfelder, E., Buchner, A., \& Lang, A.-G. (2009). Statistical power analyses using $\mathrm{G}^{*}$ Power 3.1: Tests for correlation and regression analyses. Behavior Research Methods, 41(4), 1149-1160. doi:10.3758/BRM.41.4.1149

Freire, A., Lee, K., \& Symons, L. A. (2000). The face-inversion effect as a deficit in the encoding of configural information: Direct evidence. Perception, 29(2), 159-170. doi:10.1068/p3012

Fukuda, K., Vogel, E., Mayr, U., \& Awh, E. (2010). Quantity, not quality: The relationship between fluid intelligence and working memory capacity. Psychonomic Bulletin \& Review, 17(5), 673-679. doi:10.3758/17.5.673

Gao, Z., \& Bentin, S. (2011). Coarse-to-fine encoding of spatial frequency information into visual short-term memory for faces but impartial decay. Journal of Experimental Psychology: Human Perception and Performance, 37(4), 1051-1064. doi:10.1037/a0023091

Gauthier, I., Tarr, M. J., Anderson, A. W., Skudlarski, P., \& Gore, J. C. (1999). Activation of the middle fusiform "face area" increases with expertise in recognizing novel objects. Nature Neuroscience, 2(6), 568-573. doi:10.1038/9224

Granic, I., Lobel, A., \& Engels, R. C. M. E. (2014). The benefits of playing video games. 69(1), 66-78. doi:10.1037/a0034857

Green, C. S., \& Bavelier, D. (2012). Learning, attentional control, and action video games. Current Biology, 22(6), R197-R206. doi:10.1016/j.cub.2012.02.012
Grill-Spector, K., Knouf, N., \& Kanwisher, N. (2004). The fusiform face area subserves face perception, not generic within-category identification. Nature Neuroscience, 7(5), 555-562. doi:10.1038/nn1224

Huang, L. (2011). Familiarity does not aid access to features. Psychonomic Bulletin \& Review, 18(2), 278-286. doi:10.3758 /s13423-011-0052-6

Jackson, M. C., \& Raymond, J. E. (2008). Familiarity enhances visual working memory for faces. Journal of Experimental Psychology: Human Perception and Performance, 34(3), 556-568. doi:10.1037 /0096-1523.34.3.556

James, T. W., \& James, K. H. (2013). Expert individuation of objects increases activation in the fusiform face area of children. NeuroImage, 67, 182-192. doi:10.1016/j.neuroimage.2012.11.007

Jonides, J., Lewis, R. L., Nee, D. E., Lustig, C. A., Berman, M. G., \& Moore, K. S. (2008). The mind and brain of short-term memory. Annual Review of Psychology, 59, 193-224. doi:10.1146/annurev. psych.59.103006.093615

Konkle, T., Brady, T. F., Alvarez, G. A., \& Oliva, A. (2010). Conceptual distinctiveness supports detailed visual long-term memory for realworld objects. Journal of Experimental Psychology: General, 139(3), 558-578. doi:10.1037/a0019165

Kuhn, S., \& Gallinat, J. (2014). Amount of lifetime video gaming is positively associated with entorhinal, hippocampal and occipital volume. Molecular Psychiatry, 19(7), 842-847. doi:10.1038 /mp.2013.100

Lagarias, J. C., Reeds, J. A., Wright, M. H., \& Wright, P. E. (1998). Convergence properties of the Nelder-Mead simplex method in low dimensions. SIAM Journal on Optimization, 9(1), 112-147. doi: $10.1137 / \mathrm{S} 1052623496303470$

Liu, C. H., \& Chaudhuri, A. (2000). Recognition of unfamiliar faces: Three kinds of effects. Trends in Cognitive Sciences, 4(12), 445 446. doi:10.1016/S1364-6613(00)01558-8

Lorenc, E. S., Pratte, M. S., Angeloni, C. F., \& Tong, F. (2014). Expertise for upright faces improves the precision but not the capacity of visual working memory. Attention, Perception \& Psychophysics, 76(7), 1975-1984. doi:10.3758/s13414-014-0653-z

Luck, S. J. (2008). Visual short-term memory. In S. J. Luck \& A. Hollingworth (Eds.), Visual memory (pp. 43-85). New York: Oxford University Press.

McKone, E., Kanwisher, N., \& Duchaine, B. C. (2007). Can generic expertise explain special processing for faces? Trends in Cognitive Sciences, 11(1), 8-15. doi:10.1016/j.tics.2006.11.002

Meng, X. L., Rosenthal, R., \& Rubin, D. B. (1992). Comparing correlated correlation coefficients. Psychological Bulletin, 111(1), 172-175. doi:10.1037/0033-2909.111.1.172

Moore, C. D., Cohen, M. X., \& Ranganath, C. (2006). Neural mechanisms of expert skills in visual working memory. Journal of Neuroscience, 26(43), 11187-11196. doi:10.1523 /JNEUROSCI.1873-06.2006

Morey, R. D. (2008). Confidence intervals from normalized data: A correction to Cousineau (2005). Tutorial in Quantitative Methods for Psychology, 4(2), 61-64.

Oberauer, K., \& Lange, E. B. (2009). Activation and binding in verbal working memory: A dual-process model for the recognition of nonwords. Cognitive Psychology, 58(1), 102-136. doi:10.1016/j. cogpsych.2008.05.003

Olson, I. R., \& Jiang, Y. (2004). Visual short-term memory is not improved by training. Memory \& Cognition, 32(8), 1326-1332.

Olson, I. R., Jiang, Y., \& Moore, K. S. (2005). Associative learning improves visual working memory performance. Journal of Experimental Psychology: Human Perception and Performance, 31(5), 889-900. doi:10.1037/0096-1523.31.5.889

Olsson, H., \& Poom, L. (2005). Visual memory needs categories. Proceedings of the National Academy of Sciences of the United States of American, 102(24), 8776-8780. doi:10.1073 /pnas.0500810102 
Parks, C. M., \& Yonelinas, A. P. (2007). Moving beyond pure signaldetection models: Comment on Wixted (2007). Psychological Review, 114(1), 188-201. doi:10.1037/0033-295X.114.1.188

Pashler, H. (1988). Familiarity and visual change detection. Perception \& Psychophysics, 44(4), 369-378.

Ranganath, C. (2004). Inferior temporal, prefrontal, and hippocampal contributions to visual working memory maintenance and associative memory retrieval. Journal of Neuroscience, 24(16), 3917-3925. doi:10.1523/JNEUROSCI.5053-03.2004

Rezlescu, C., Barton, J. J. S., Pitcher, D., \& Duchaine, B. (2014). Normal acquisition of expertise with greebles in two cases of acquired prosopagnosia. Proceedings of the National Academy of Sciences of the United States of America, 111(14), 5123-5128. doi:10.1073 /pnas.1317125111

Rouder, J. N., Morey, R. D., Cowan, N., Zwilling, C. E., Morey, C. C., \& Pratte, M. S. (2008). An assessment of fixed-capacity models of visual working memory. Proceedings of the National Academy of Sciences of the United States of America, 105(16), 5975-5979. doi:10.1073/pnas.0711295105

Scolari, M., Vogel, E. K., \& Awh, E. (2008). Perceptual expertise enhances the resolution but not the number of representations in working memory. Psychonomic Bulletin \& Review, 15(1), 215-222. doi:10.3758/PBR.15.1.215

Sekuler, A. B., Gaspar, C. M., Gold, J. M., \& Bennett, P. J. (2004). Inversion leads to quantitative, not qualitative, changes in face processing. Current Biology, 14(5), 391-396. doi:10.1016/j. cub.2004.02.028

Sørensen, T. A., \& Kyllingsbæk, S. (2012). Short-term storage capacity for visual objects depends on expertise. Acta Psychologica, 140(2), 158-163. doi:10.1016/j.actpsy.2012.04.004
Thorn, A., \& Page, M. (2009). Interactions between short-term and long-term memory in the verbal domain. New York: Psychological Press.

Vogel, E. K., Woodman, G. F., \& Luck, S. J. (2006). The time course of consolidation in visual working memory. Journal of Experimental Psychology: Human Perception and Performance, 32(6), 14361451. doi:10.1037/0096-1523.32.6.1436

Wagar, B. M., \& Dixon, M. J. (2005). Past experience influences object representation in working memory. Brain and Cognition, 57(3), 248-256. doi:10.1016/j.bandc.2004.08.054

Wagenmakers, E.-J., \& Farrell, S. (2004). AIC model selection using Akaike weights. Psychonomic Bulletin \& Review, 11(1), 192-196. doi:10.3758/BF03206482

Wickens, T. D. (2001). Elementary signal detection theory. New York: Oxford University Press Oxford.

Wixted, J. T. (2007). Dual-process theory and signal-detection theory of recognition memory. Psychological Review, 114(1), 152-176. doi:10.1037/0033-295X.114.1.152

Xie, W., \& Zhang, W. (2016a). Experimental dissociations of STM quantity and quality using receiver operation characteristics (ROCs). Manuscript in preparation.

Xie, W., \& Zhang, W. (2016b). Familiarity speeds up visual short-term memory consolidation. Journal of Experimental Psychology: Human Perception and Performance. doi:10.1037/xhp0000355

Yonelinas, A. P., \& Parks, C. M. (2007). Receiver operating characteristics (ROCs) in recognition memory: A review. Psychological Bulletin, 133(5), 800-832. doi:10.1037/0033-2909.133.5.800

Zhang, W., \& Luck, S. J. (2008). Discrete fixed-resolution representations in visual working memory. Nature, 453(7192), 233-235. doi:10.1038/nature06860 